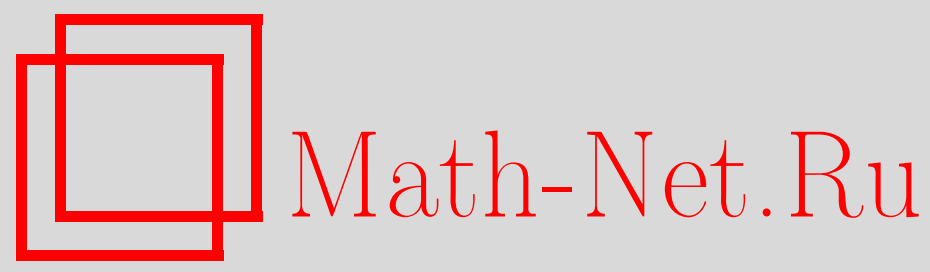

В. Д. Седых, Особенности коранга 1 устойчивых гладких отображений и особые касательные гиперплоскости пространственной кривой, Матем. заметки, 2005, том 78, выпуск $3,413-427$

DOI: https://doi.org/10.4213/mzm2598

Использование Общероссийского математического портала Math-Net.Ru подразумевает, что вы прочитали и согласны с пользовательским соглашением http://www . mathnet.ru/rus/agreement

Параметры загрузки:

IP : 54.198 .187 .58

26 апреля 2023 г., $17: 18: 24$

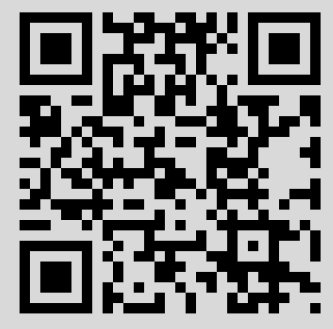




\title{
ОСОБЕННОСТИ КОРАНГА 1 УСТОЙЧИВЫХ ГЛАДКИХ ОТОБРАЖЕНИЙ И ОСОБЫЕ КАСАТЕЛЬНЫЕ ГИПЕРПЛОСКОСТИ ПРОСТРАНСТВЕННОЙ КРИВОЙ
}

\section{В. Д. Седых}

\begin{abstract}
Пусть $\gamma$ - гладкая замкнутая кривая общего положения в $\mathbb{R} P^{3}$. Обозначим через $C$ число ее точек уплощения, а через $T$ - число плоскостей, касающихся $\gamma$ в трех различных точках. Рассмотрим соприкасающиеся плоскости кривой $\gamma$ в точках уплощения. Пусть $N$ - общее число точек, в которых $\gamma$ трансверсально пересекает эти соприкасающиеся плоскости. Тогда $T \equiv[N+\theta(\gamma) C] / 2(\bmod 2)$, где $\theta(\gamma)$ - число нестягиваемых компонент кривой $\gamma$. Это сравнение обобщает известную теорему Фридмана о том, что если гладкая связная замкнутая кривая общего положения в $\mathbb{R}^{3}$ не имеет точек уплощения, то число ее тройных касательных плоскостей четно. Мы приводим также многомерные аналоги указанной формулы и показьваем, что эти результаты являются следствиями некоторых общих фактов о топологии особенностей коранга 1 устойчивых гладких отображений многообразий одинаковой размерности.

Библиограффия: 13 названий.
\end{abstract}

1. Введение. Рассмотрим $C^{\infty}$-гладкую замкнутую кривую $\gamma$ в $n$-мерном вещественном проективном пространстве $\mathbb{R} P^{n}$. Эта кривая может иметь несколько связных компонент, каждая из которых задается гладким отображением $S^{1} \rightarrow \mathbb{R} P^{n}$. Число нестягиваемых компонент кривой $\gamma$ обозначим через $\theta(\gamma)$.

Напомним, что соприкасающейся гиперплоскостью кривой $\gamma$ в точке $x$ назьвается любая гиперплоскость в $\mathbb{R} P^{n}$, которую кривая пересекает в $x$ с кратностью, не меньшей $n$. Любая кривая имеет, по меньшей мере, одну соприкасающуюся гиперплоскость в каждой своей точке. Точка, в которой соприкасающаяся гиперплоскость кривой не единственна, называется точкой вырождения. Точка кривой в $\mathbb{R} P^{n}$ назьвается точкой уплощения, если кратность пересечения кривой со своей соприкасающейся гиперплоскостью в этой точке больше $n$.

Снабдим $C^{\infty}$-топологией пространство $W$ всех гладких замкнутых кривых в $\mathbb{R} P^{n}$. Мы будем говорить, что данное утверждение справедливо для кривой общего положения, если существует открытое всюду плотное подмножество в пространстве $W$ такое, что указанное утверждение верно для всех кривых из этого подмножества.

Кривая $\gamma$ общего положения в $\mathbb{R} P^{n}$ не имеет точек вырождения, иммерсирована, если $n=2$, и вложена при $n>2$. Число $\mathscr{S}_{\gamma}^{0}\left(A_{n}\right)$ ее точек уплощения конечно и

Работа выполнена при частичной поддержке Российского фонда фундаментальных исследований, грант № 05-01-00104. 
$\mathscr{S}_{\gamma}^{0}\left(A_{n}\right) \equiv(n+1) \theta(\gamma)(\bmod 2)$. Кривая $\gamma$ может пересекать любую гиперплоскость только в конечном числе точек (взятых с учетом кратностей). Она может касаться гиперплоскости не более, чем в $n$ геометрически различных точках, причем сумма кратностей этих касаний не больше $n$. Число $\mathscr{S}_{\gamma}^{0}\left(n A_{1}\right)$ всех $n$-касательны $x$ гиперплоскостей кривой $\gamma$ (которые касаются ее в $n$ различных точках) конечно.

Рассмотрим случай $n=3$. Пусть $T=\mathscr{S}_{\gamma}^{0}\left(3 A_{1}\right), C=\mathscr{S}_{\gamma}^{0}\left(A_{3}\right)$ и $N$ - общее число точек трансверсального пересечения кривой $\gamma$ со своими соприкасающимися плоскостями в точках уплошения. Тогда $T \equiv N / 2(\bmod 2)$ для любой гладкой связной замкнутой кривой $\gamma$ общего положения в аффинном пространстве $\mathbb{R}^{3} \subset \mathbb{R} P^{3}$.

Эта формула была получена в [1] как обобщение теоремы [2], согласно которой число тройных касательных плоскостей гладкой связной замкнутой кривой общего положения и без точек уплощения в $\mathbb{R}^{3}$ четно. В настоящей статье мы обобщаем эти результаты на произвольные гладкие замкнутые кривые общего положения в $\mathbb{R} P^{3}$.

ТЕОРема 1.1. Для любой гладкой замкнутой кривой $\gamma$ общего положсения в $\mathbb{R} P^{3}$

$$
T \equiv \frac{N+\theta(\gamma) C}{2}(\bmod 2)
$$

В частности, если кривая $\gamma$ не имеет точек уплощения, то число ее тройных касательных плоскостей четно.

В работе приводится также многомерный аналог теоремы 1.1. А именно, рассмотрим гиперплоскости в $\mathbb{R} P^{n}$, которые касаются данной кривой $\gamma$ в $n-2$ точках с кратностями 1 (в $n-3$ точках) и 3 . Пусть $\mathscr{S}_{\gamma}^{0}\left(A_{3}+(n-3) A_{1}\right)$ - число всех таких гиперплоскостей, а $\mathscr{S}_{\gamma}^{1}\left(A_{3}+(n-3) A_{1}\right)$ - число гиперплоскостей (указанного типа), у каждой из которых число точек трансверсального пересечения с кривой $\gamma$ сравнимо с $2 n-\theta(\gamma)$ по модулю 4 . У замкнутой кривой $\gamma$ общего положения числа $\mathscr{S}_{\gamma}^{0}\left(A_{3}+(n-3) A_{1}\right)$ и $\mathscr{S}_{\gamma}^{1}\left(A_{3}+(n-3) A_{1}\right)$ конечны. Если $n=3$, то число $\mathscr{S}_{\gamma}^{1}\left(A_{3}\right)$ сравнимо с $[N+\theta(\gamma) C] / 2$ по модулю 2 .

ТЕОРемА 1.2. Для любой гладкой замкнутой кривой $\gamma$ общего положсения в пространстве $\mathbb{R} P^{n}$ нечетной размерности $n \geqslant 3$

$$
\mathscr{S}_{\gamma}^{0}\left(n A_{1}\right) \equiv \mathscr{S}_{\gamma}^{1}\left(A_{3}+(n-3) A_{1}\right)(\bmod 2)
$$

В частности, если $\mathscr{S}_{\gamma}^{0}\left(A_{3}+(n-3) A_{1}\right)=0$, то число $n$-касательных гиперплоскостей кривой $\gamma$ четно.

Формула (1) имеет и другие многомерные обобщения. Приведем еще одно.

Пусть $n \geqslant 3$ нечетно. Обозначим через $\mathscr{S}_{\gamma}^{0}\left(2 A_{(n-1) / 2}+A_{1}\right)$ число гиперплоскостей, которые касаются кривой $\gamma \subset \mathbb{R} P^{n}$ в трех точках с кратностями $(n-1) / 2$ (в двух точках) и 1 . Через $\mathscr{S}_{\gamma}^{1}\left(A_{n}\right)$ обозначим число точек уплощения кривой $\gamma$, удовлетворяющих следующему условию: если $\pi$ - соприкасающаяся гиперплоскость кривой в такой точке, то число точек трансверсального пересечения $\gamma$ с $\pi$ сравнимо с $1-n-\theta(\gamma)$ по модулю 4. У замкнутой кривой $\gamma$ общего положения, числа $\mathscr{S}_{\gamma}^{0}\left(2 A_{(n-1) / 2}+A_{1}\right)$ и $\mathscr{S}_{\gamma}^{1}\left(A_{n}\right)$ конечны. 
ТЕОРемА 1.3. Для любой гладкой замкнутой кривой $\gamma$ общего положсения в пространстве $\mathbb{R} P^{n}$ нечетной размерности $n \geqslant 3$

$$
\mathscr{S}_{\gamma}^{0}\left(2 A_{(n-1) / 2}+A_{1}\right) \equiv \mathscr{S}_{\gamma}^{1}\left(A_{n}\right)(\bmod 2) .
$$

В частности, если кривая $\gamma$ не имеет точек уплощения, то число $\mathscr{S}_{\gamma}^{0}\left(2 A_{(n-1) / 2}+\right.$ $A_{1}$ ) четно.

Все эти результаты являются следствиями одной общей формулы, которая описывает глобальные свойства проективных кривых (см. теорему 5.2 ниже). С другой стороны, эта формула является следствием некоторых общих фактов о топологии особенностей коранга 1 устойчивых гладких отображений многообразий, имеющих одинаковую размерность. Мы начнем с изучения этих особенностей.

ЗАмЕчАниЕ 1.4. Фридман высказал в [2] гипотезу о том, что если гладкая связная замкнутая кривая общего положения в $\mathbb{R}^{n}$, где $n>3$, не имеет точек уплощения, то число ее $n$-касательных гиперплоскостей четно. Я не обнаружил контрпример к этой гипотезе в литературе. Но формула (2) показьвает, что такой контрпример весьма вероятен.

ЗАмЕчание 1.5. Мнене удалось получить каких-либо ограничений на четность числа $n$-касательных гиперплоскостей гладкой замкнутой кривой общего положения в четномерном пространстве $\mathbb{R} P^{n}$.

2. Особенности коранга 1 устойчивых гладких отображений многообразий одинаковой размерности. Пусть $M$ и $N$-гладкие $n$-мерные многообразия, причем $M$ замкнуто (компактное без края). Рассмотрим гладкое отображение $f: M \rightarrow N$.

ОПРЕДЕЛЕНИЕ 2.1. Отображение $f$ называется отображением коранга $\leqslant 1$, если для любого $x \in M$ размерность ядра производной $f_{* x}: T_{x} M \rightarrow T_{f(x)} N$ не больше 1 .

Снабдим $C^{\infty}$-топологией пространство всех гладких отображений $M$ в $N$. Отображение $f$ назьвается устойчивым, если для любого отображения $\tilde{f}: M \rightarrow N$, достаточно близкого к $f$, существуют диффеоморфизмы $g: M \rightarrow M$ и $h: N \rightarrow N$ такие, что $h \circ f=\tilde{f} \circ g$.

Устойчивые отображения образуют открытое подмножество в пространстве всех отображений $M$ в $N$. Они плотны в пространстве отображений коранга $\leqslant 1$.

Пусть $f$ - устойчивое гладкое отображение коранга $\leqslant 1$. Тогда оно имеет только особенности типов $A_{\mu}$ с целыми неотрицательными $\mu \leqslant n$ (см. [3]).

ОПРедЕЛЕниЕ 2.2. Отображение $f$ имеет особенность типа $A_{\mu}$ в точке $x \in M$, если существуют локальные координаты на многообразиях $M$ и $N$ такие, что росток $(f, x)$ отображения $f$ в точке $x$ задается формулой

$$
(f, x):\left(\mathbb{R}^{n}, 0\right) \rightarrow\left(\mathbb{R}^{n}, 0\right), \quad(t, \bar{\lambda}) \mapsto(S(t, \bar{\lambda}), \bar{\lambda}),
$$

где

$$
\bar{\lambda}=\left(\lambda_{1}, \ldots, \lambda_{n-1}\right), \quad S(t, \bar{\lambda})=t^{\mu+1}+\lambda_{\mu-1} t^{\mu-1}+\cdots+\lambda_{1} t
$$

Число $\mu$ назьвается показателем вырожденности особенности типа $A_{\mu}$. Множество $A_{\mu}^{f}$ точек $x \in M$, в которых $f$ имеет особенность типа $A_{\mu}$, является гладким 
подмногообразием коразмерности $\mu$ в $M$. Образ этого многообразия относительно отображения $f$ является иммерсированным подмногообразием той же коразмерности в $N$. Многообразие $A_{\mu}^{f}$ и его образ, вообще говоря, незамкнуты и несвязны.

Пусть $\mathbb{A}$ - свободная абелева полугрупша по сложению, образующими которой служат символы $A_{0}, A_{1}, \ldots, A_{\mu}, \ldots$ Рассмотрим произвольньй ненулевой элемент $\mathscr{A}=$ $A_{\mu_{1}}+\cdots+A_{\mu_{p}} \in \mathbb{A}$.

ОПРЕДЕЛЕНИЕ 2.3. Отображение $f$ имеет мультиособенность типа $\mathscr{A}$ в точке $y \in N$, если:

1) полный прообраз $f^{-1}(y)$ точки $y$ при отображении $f$ состоит из $p$ попарно различных точек;

2 ) сушествует порядок $x_{1}, \ldots, x_{p}$ точек из $f^{-1}(y)$ такой, что $f$ имеет в этих точках особенности типов $A_{\mu_{1}}, \ldots, A_{\mu_{p}}$, соответственно;

3 ) образы при отображении $f$ ростков $\left(A_{\mu_{i}}^{f}, x_{i}\right)$ в $x_{i}$ многообразий $A_{\mu_{i}}^{f}, i=1, \ldots, p$, пересекаются трансверсально в точке $y$.

ЗАмЕчАниЕ 2.4. Мы будем также говорить, что в точках из дополнения $N \backslash f(M)$ отображение $f$ имеет мультиособенность типа 0 (нуль полугрупшы $\mathbb{A}$ ).

Числа $\mu_{1}, \ldots, \mu_{p}$ назьваются показателями вырожденности мультиособенностей типа $\mathscr{A}$ отображения $f$. Числа

$$
\operatorname{codim} \mathscr{A}=\mu_{1}+\cdots+\mu_{p} \quad \text { и } \quad \operatorname{deg} \mathscr{A}=\operatorname{codim} \mathscr{A}+p
$$

назьваются коразмерностью и степенью этих мультиособенностей, соответственно. Если $\mathscr{A}=0$, то codim $\mathscr{A}=\operatorname{deg} \mathscr{A}=0$.

Устойчивое гладкое отображение $f: M \rightarrow N$ коранга $\leqslant 1$ может иметь мультиособенности только типов $\mathscr{A} \in \mathbb{A}$. Коразмерность мультиособенности не превышает размерности $n$ объемлющего многообразия $N$. Степень мультиособенности ограничена максимумом $d_{f}$ числа точек в полном прообразе $f^{-1}(y)$ по всем $y \in N$.

Рассмотрим множество $\mathscr{A}_{f}$ точек $y \in N$, в которых $f$ имеет мультиособенность типа $\mathscr{A}$. Это множество является гладким подмногообразием коразмерности $\operatorname{codim} \mathscr{A}$ в $N$ (незамкнутым и несвязным, вообще говоря).

ОПРЕДЕЛЕНИЕ 2.5. Многообразие $\mathscr{A}_{f}$ назьвается многообразием мультиособенностей типа $\mathscr{A}$ отображения $f$.

Пусть $\mathscr{F}_{f}$ - множество критических значений отображения $f$. Если $f$ - устойчивое гладкое отображение коранга $\leqslant 1$, то $\mathscr{F}_{f}$ - устойчивьй компактный фронт в многообразии $N$, имеющий лишь особенности коранга 1 (см. [4]). Эти особенности классифицируются по элементам свободной абелевой полугруппы $\mathbb{A}_{+} \subset \mathbb{A}$ по сложению, образующими которой служат символы $A_{1}, A_{2}, \ldots, A_{\mu}, \ldots$ А именно, фронт $\mathscr{F}_{f}$ имеет особенность типа $\mathscr{A} \in \mathbb{A}_{+}$в точке $y \in N$, если и только если существует целое $k \geqslant 0$ такое, что отображение $f$ имеет в $y$ мультиособенность типа $\mathscr{A}+k A_{0} \in \mathbb{A}$.

Как известно, особенности любого устойчивого компактного фронта $\mathscr{F}$ в гладком многообразии удовлетворяют многочисленным условиям сосуществования (см., например, [5]-[7]). В случае $\mathscr{F}=\mathscr{F}_{f}$ эти условия определяют некоторые ограничения на топологию мультиособенностей отображения $f$. Но имеются и другие ограничения, которые определяются тем, что фронт $\mathscr{F}_{f}$ получен при помощи глобально определенного отображения. Некоторые из таких ограничений найдены ниже. 
3. Об эйлеровых характеристиках многообразий мультиособенностей. Пусть $M$ и $N$ - гладкие замкнутые $n$-мерные многообразия и $f: M \rightarrow N$ - устойчивое гладкое отображение коранга $\leqslant 1$. Зафиксируем произвольный элемент $\mathscr{A} \in \mathbb{A}$ и через $\overline{\mathscr{A}}$ обозначим минимальное подмножество в $\mathbb{A}$ такое, что

1) $\mathscr{A} \in \overline{\mathscr{A}}$;

2) если $X=A_{\nu_{1}}+\cdots+A_{\nu_{q}} \in \overline{\mathscr{A}}$, то

$$
X+A_{1} \in \overline{\mathscr{A}}, \quad X-A_{\nu_{i}}-A_{\nu_{j}}+A_{\nu_{i}+\nu_{j}+1} \in \overline{\mathscr{A}}
$$

для всех $1 \leqslant i<j \leqslant q$.

Рассмотрим замькание $\overline{\mathscr{A}_{f}}$ многообразия $\mathscr{A}_{f}$ мультиособенностей типа $\mathscr{A}$ отображения $f$ в объемлющем многообразии $N$. Легко видеть, что множество $\overline{\mathscr{A}_{f}}$ компактно и является объединением многообразий $X_{f}$ мультиособенностей типов $X \in \overline{\mathscr{A}}$. Разбиение множества $\overline{\mathscr{A}_{f}}$ на связные компоненты этих многообразий является конечной $C^{\infty}$-стратификацией Уитни.

Пусть $\partial(\mathscr{A})=\overline{\mathscr{A}} \backslash \mathscr{A} \subset \mathbb{A}$. Предположим, что отображение $f$ имеет мультиособенность типа $X \in \partial(\mathscr{A})$ в данной точке $y \in N$ и положим $c=\operatorname{codim} X$. Выберем окрестность $U$ начала координат 0 в $\mathbb{R}^{c}$ и гладкое вложение $\tau: U \rightarrow N$ так, что $\tau(0)=y$, а подмногообразие $\tau(U) \subset N$ трансверсально многообразию $X_{f}$ в точке $y$. Через $S_{\varepsilon}$ обозначим $(c-1)$-мерную сферу в $\mathbb{R}^{c}$ радиуса $\varepsilon>0$ с центром в 0 . Легко видеть, что существует $\varepsilon_{0}(f, y, \tau)>0$ такое, что число $J_{\mathscr{A}}(X)$ связных компонент пересечения $\tau\left(S_{\varepsilon}\right) \cap \mathscr{A}_{f}$ не зависит от $f, y, \tau$ и $\varepsilon$, если $\varepsilon<\varepsilon_{0}(f, y, \tau)$.

ОПРЕДЕЛЕНИЕ 3.1. Число $J_{\mathscr{A}}(X)$ называется индексом примыкания мультиособенности типа $X$ отображения $f$ к мультиособенностям типа $\mathscr{A}$.

ЗАмечание 3.2. Число $J_{\mathscr{A}}(X)=0$, если $X \notin \partial \mathscr{A}$.

Индекс $J_{\mathscr{A}}(X)$ можно вычислить следуюшим образом. Пусть $\mathscr{A}=A_{\mu_{1}}+\cdots+A_{\mu_{p}}$, $X=A_{\nu_{1}}+\cdots+A_{\nu_{q}}$ и $d=\operatorname{deg} X$. Возьмем пространство $\mathbb{R}^{d}$ упорядоченных наборов $\left(P_{1}, \ldots, P_{q}\right)$ многочленов $P_{i}=t^{\nu_{i}+1}+\lambda_{\nu_{i}}^{i} t^{\nu_{i}}+\cdots+\lambda_{1}^{i} t+\lambda_{0}^{i}$ от $t \in \mathbb{R}$ с вещественными коэффициентами. Через $\left[P_{i}\right]_{\mathbb{R}}$ обозначим неупорядоченньй набор кратностей вещественных корней многочлена $P_{i}$. Два набора многочленов әквивалентны, если найдется путь $\left(P_{1}^{s}, \ldots, P_{q}^{s}\right) \in \mathbb{R}^{d}, s \in[0,1]$, связьвающий эти наборы, такой, что набор $\left(\left[P_{1}^{s}\right]_{\mathbb{R}}, \ldots,\left[P_{q}^{s}\right]_{\mathbb{R}}\right)$ одинаков для всех $s$.

ПРЕДЛОЖЕНИЕ 3.3. Индекс $J_{\mathscr{A}}(X)$ равен числу классов әквивалентности наборов $\left(P_{1}, \ldots, P_{q}\right) \in \mathbb{R}^{d}$ многочленов таких, что дизбюнктное оббединение наборов $\left[P_{1}\right]_{\mathbb{R}}, \ldots,\left[P_{q}\right]_{\mathbb{R}}$ является неупорядоченным множеством чисел $\mu_{1}+1, \ldots, \mu_{p}+1$.

Это непосредственно вытекает из определения индекса $J_{\mathscr{A}}(X)$.

ПримеР 3.4. $J_{A_{1}+2 A_{0}}\left(A_{3}+A_{1}\right)=5$. В самом деле, возьмем пространство $\mathbb{R}^{6}$ упорядоченных пар $\left(P_{1}, P_{2}\right)$ многочленов $P_{1}=t^{2}+\lambda_{1}^{1} t+\lambda_{0}^{1}$ и $P_{2}=t^{4}+\lambda_{3}^{2} t^{3}+\lambda_{2}^{2} t^{2}+\lambda_{1}^{2} t+\lambda_{0}^{2}$. Имеется ровно пять классов эквивалентности пар $\left(P_{1}, P_{2}\right)$ таких, что дизъюнктное объединение наборов $\left[P_{1}\right]_{\mathbb{R}},\left[P_{2}\right]_{\mathbb{R}}$ совпадает с неупорядоченным набором чисел $1,1,2$. Представители этих классов изображены на рис. 1. 

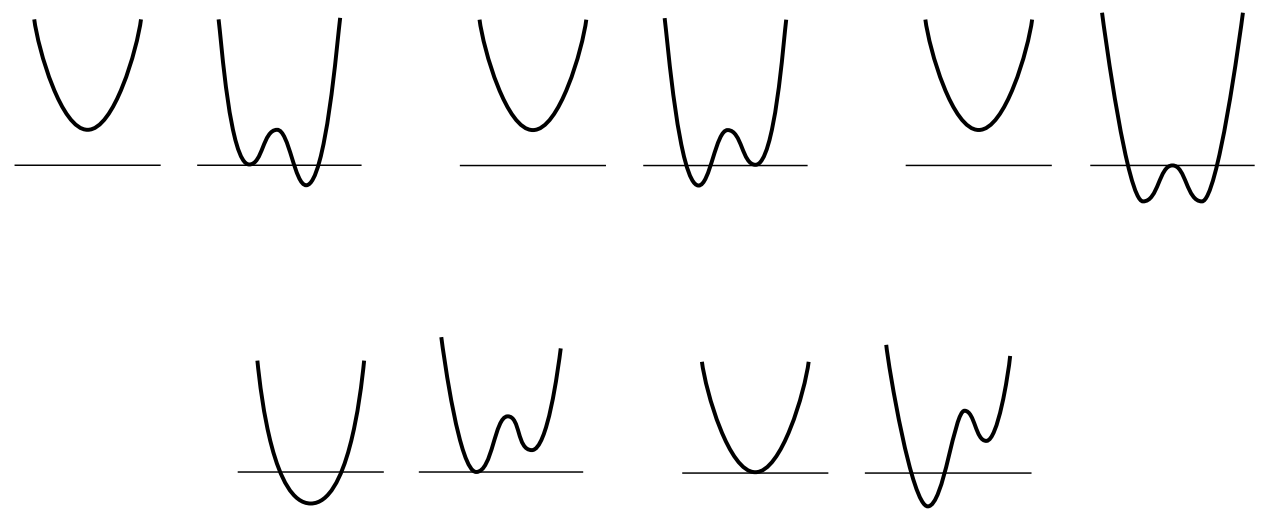

Рис. 1

Пусть $d_{f}$ - максимум числа точек в полном прообразе $f^{-1}(y)$ по всем $y \in N$. Зафиксируем отображение $f$ и рассмотрим пространство иепей (конечных линейных комбинаций) вида

$$
\sigma=\sum_{\mathscr{A} \in \mathbb{A}: \operatorname{deg} \mathscr{A} \leqslant d_{f}} \alpha_{\mathscr{A}} \cdot \mathscr{A}_{f}
$$

с цельми коэффициентами $\alpha_{\mathscr{A}}$. Сложение цепей (как линейных комбинаций) превращает это пространство в свободную абелеву групш, порожденную многообразиями $\mathscr{A}_{f}$ мультиособенностей всех типов $\mathscr{A} \in \mathbb{A}$, возможных при данном значении $d_{f}$. Гранииу цепи $\sigma$ определим формулой

$$
\partial_{*}(\sigma)=\sum_{\mathscr{A} \in \mathbb{A}: \operatorname{deg} \mathscr{A} \leqslant d_{f}} \alpha_{\mathscr{A}} \cdot \partial_{*}\left(\mathscr{A}_{f}\right),
$$

где

$$
\partial_{*}\left(\mathscr{A}_{f}\right)=\sum_{X \in \partial \mathscr{A}: \operatorname{deg} X \leqslant d_{f}}(-1)^{\operatorname{codim} X} J_{\mathscr{A}}(X) \cdot X_{f}
$$

Пусть $\chi\left(\mathscr{A}_{f}\right)$ - (топологическая) эйлерова характеристика многообразия $\mathscr{A}_{f}$ (альтернированная сумма чисел Бетти групп гомологий с компактными носителями). Рассмотрим коцепь

$$
\chi(\sigma)=\sum_{\mathscr{A} \in \mathbb{A}: \operatorname{deg} \mathscr{A} \leqslant d_{f}} \alpha \mathscr{A} \chi\left(\mathscr{A}_{f}\right) .
$$

Кограницу $\partial^{*}(\chi)$ этой коцепи определим как обычно:

$$
\left(\partial^{*}(\chi)\right)(\sigma)=\sum_{\mathscr{A} \in \mathbb{A}: \operatorname{deg} \mathscr{A} \leqslant d_{f}} \alpha_{\mathscr{A}}\left(\partial^{*}(\chi)\right)\left(\mathscr{A}_{f}\right),
$$

где $\left(\partial^{*}(\chi)\right)\left(\mathscr{A}_{f}\right)=\chi\left(\partial_{*}\left(\mathscr{A}_{f}\right)\right)$.

Следующее утверждение неявно содержится в [8].

ПРЕДЛОЖЕНИЕ 3.5. ДЛя любого $\mathscr{A} \in \mathbb{A}$

$$
\sum_{X \in \partial \mathscr{A}}(-1)^{\operatorname{codim} X} J_{\mathscr{A}}(X) \chi\left(X_{f}\right)= \begin{cases}2(-1)^{n} \chi\left(\mathscr{A}_{f}\right), & \text { если } \mathscr{A}_{f} \text { нечетномерное } \\ 0, & \text { если } \mathscr{A}_{f} \text { четномерное. }\end{cases}
$$


ДокАЗАТЕЛЬСтво. Зафиксируем произвольньй элемент $\mathscr{A} \in \mathbb{A}$ и рассмотрим указанную выше $C^{\infty}$-стратификацию множества $\overline{\mathscr{A}_{f}}$. Возьмем те страты этой стратификации, которые не являются связными компонентами многообразия $\mathscr{A}_{f}$. Согласно [9], [10] существует система трубчатых окрестностей этих стратов в многообразии $N$ такая, что объединение $\mathscr{O}$ этих окрестностей удовлетворяет следующим условиям:

1) множество $\mathscr{A}_{f} \backslash \mathscr{O}$ является компактным $C^{0}$-многообразием с краем $\partial\left(\mathscr{A}_{f} \backslash \mathscr{O}\right)$;

2) $\mathscr{A}_{f} \backslash \mathscr{O}$ гомотопически эквивалентно $\mathscr{A}_{f}$ и гомеоморфно гладкому многообразию (с краем);

3) $\chi\left(\partial\left(\mathscr{A}_{f} \backslash \mathscr{O}\right)\right)=(-1)^{n} \sum_{X \in \partial \mathscr{A}}(-1)^{\operatorname{codim} X} J_{\mathscr{A}}(X) \chi\left(X_{f}\right)$.

Теперь предложение 3.5 следует из того факта, что

$$
\chi\left(\partial\left(\mathscr{A}_{f} \backslash \mathscr{O}\right)\right)= \begin{cases}2 \chi\left(\mathscr{A}_{f} \backslash \mathscr{O}\right), & \text { если } \mathscr{A}_{f} \text { нечетномерное } \\ 0, & \text { если } \mathscr{A}_{f} \text { четномерное. }\end{cases}
$$

СлЕДСТВИЕ 3.6. Пусть $M$ и $N$ - гладкие замкнутые $n$-мерные многообразия $и$ $f: M \rightarrow N-$ устойчивое гладкое отображение коранга $\leqslant 1$. Тогда $\chi\left(\partial_{*}\left(\mathscr{A}_{f}\right)\right) \equiv 0$ $(\bmod 2)$ для любого $\mathscr{A} \in \mathbb{A}$, т.е. коиепь $\chi$ является кочиклом по модулю 2.

ЗАмЕЧАнИЕ 3.7. Из предложения 3.5 также следует, что эйлерова характеристика $\chi\left(\mathscr{A}_{f}\right)$ нечетномерного многообразия $\mathscr{A}_{f}$ мультиособенностей любого типа $\mathscr{A} \in \mathbb{A}$ у каждого отображения $f$ рассматриваемого вида является линейной комбинацией эйлеровых характеристик $\chi\left(X_{f}\right)$ четномерных многообразий $X_{f}$, образованных мультиособенностями типов $X \in \mathbb{A}$ таких, что $\operatorname{codim} X \geqslant \operatorname{codim} \mathscr{A}+1$. Эта комбинация универсальна в том смысле, что каждый ее коэффициент зависит только от типов $\mathscr{A}$ и $X$ соответствующих мультиособенностей (т.е. не зависит от $M, N$ и $f$ ). Аналогичньй факт имеет место и для устойчивых гладких отображений коранга $\leqslant 1$ в многообразие большей размерности (см. [11]).

Следующее утверждение описьвает некоторые соотношения между индексами примыкания мультиособенностей устойчивого гладкого отображения коранга $\leqslant 1$ многообразий одинаковой размерности.

ПРЕДЛОЖЕНИЕ 3.8. Для любых $\mathscr{A}, Y \in \mathbb{A} \operatorname{ma\kappa ux,~ито~} Y \in \partial \mathscr{A}$,

$$
\begin{aligned}
& \sum_{X \in \partial \mathscr{A}}(-1)^{\operatorname{codim} X} J_{\mathscr{A}}(X) J_{X}(Y) \\
& \quad= \begin{cases}2(-1)^{\operatorname{codim} Y-1} J_{\mathscr{A}}(Y), & \text { ecлu } \operatorname{codim} Y \equiv \operatorname{codim} \mathscr{A}(\bmod 2), \\
0, & \text { ecлu } \operatorname{codim} Y \equiv \operatorname{codim} \mathscr{A}+1(\bmod 2) .\end{cases}
\end{aligned}
$$

ДокАЗАтЕЛЬСтво. Пусть $m=\operatorname{codim} Y$ и $g: \mathbb{R}^{m} \rightarrow \mathbb{R}^{m}-$ устойчивое гладкое отображение коранга $\leqslant 1$, имеющее мультиособенность типа $Y$ в начале координат 0 пространства $\mathbb{R}^{m}$-образа. Рассмотрим $(m-1)$-мерную сферу $S_{\varepsilon} \subset \mathbb{R}^{m}$ радиуса $\varepsilon>0 \mathrm{c}$ центром в 0 . Если $\varepsilon$ достаточно мало, то множество $g^{-1}\left(S_{\varepsilon}\right)$ является гладким $(m-1)$ мерным подмногообразием в $\mathbb{R}^{m}$-прообразе, а сужение $\tilde{g}$ отображения $g$ на это подмногообразие является устойчивым гладким отображением коранга $\leqslant 1$. При этом $\chi\left(X_{\tilde{g}}\right)=J_{X}(Y), \chi\left(\mathscr{A}_{\tilde{g}}\right)=J_{\mathscr{A}}(Y)$ для любых $\mathscr{A}, X \in \mathbb{A}$. Остается применить предложение 3.5 к отображению $\tilde{g}$. 
СЛЕДСТВИЕ 3.9. Пусть $M u N$ - гладкие замкнутые $n$-мерные многообразия $u$ $f: M \rightarrow N-$ устойчивое гладкое отображсение коранга $\leqslant 1$. Тогда для любого $\mathscr{A} \in \mathbb{A}$

$$
\partial_{*}^{2}\left(\mathscr{A}_{f}\right)=-2 \sum_{Y} J_{\mathscr{A}}(Y) \cdot Y_{f},
$$

где сумма берется по всем $Y \in \partial \mathscr{A}$ таким, ито $\operatorname{codim} Y \equiv \operatorname{codim} \mathscr{A}(\bmod 2) u$ $\operatorname{deg} Y \leqslant d_{f}$. В частности, $\partial_{*}^{2}\left(\mathscr{A}_{f}\right) \equiv 0(\bmod 2)$.

Действительно,

$$
\begin{aligned}
\partial_{*}^{2}\left(\mathscr{A}_{f}\right) & =\sum_{X \in \partial \mathscr{A}: \operatorname{deg} X \leqslant d_{f}}(-1)^{\operatorname{codim} X} J_{\mathscr{A}}(X)\left[\sum_{Y \in \partial X: \operatorname{deg} Y \leqslant d_{f}}(-1)^{\operatorname{codim} Y} J_{X}(Y) \cdot Y_{f}\right] \\
& =\sum_{Y \in \partial \mathscr{A}: \operatorname{deg} Y \leqslant d_{f}}(-1)^{\operatorname{codim} Y}\left[\sum_{X \in \partial \mathscr{A}}(-1)^{\operatorname{codim} X} J_{\mathscr{A}}(X) J_{X}(Y)\right] \cdot Y_{f} .
\end{aligned}
$$

Поэтому указанная формула следует из предложения 3.8 .

ЗАмЕчАниЕ 3.10. Мы изучаем в этой работе только эйлеровы характеристики многообразий мультиособенностей отображения. Вопросы о классах (ко)гомологий этих многообразий - предмет дальнейшего исследования.

4. Некоторые соотношения между эйлеровыми характеристиками. Пусть $M$ и $N$ - гладкие замкнутые $n$-мерные многообразия, причем $N$ связно. Рассмотрим устойчивое гладкое отображение $f: M \rightarrow N$ коранга $\leqslant 1$. Поскольку многообразие $N$ связно, степени всех мультиособенностей отображения $f$ имеют одинаковую четность, равную четности максимума $d_{f}$ числа точек в полном прообразе $f^{-1}(y)$ по всем $y \in N$.

ОПрЕДЕЛЕНИЕ 4.1. Для любого $\mathscr{A} \in \mathbb{A}$ целое число

$$
\operatorname{ind}_{f}(\mathscr{A})=\frac{\operatorname{deg} \mathscr{A}+d_{f}}{2}
$$

назьвается индексом мультиособенности типа $\mathscr{A}$ отображения $f$.

Пусть $\mathscr{A}=A_{\mu_{1}}+\cdots+A_{\mu_{p}} \in \mathbb{A}_{+}$(т.е. $\mu_{1}, \ldots, \mu_{p}$ - целые положительные числа). Для любого целого $k \geqslant 0$ положим $\mathscr{A}^{k}=\mathscr{A}+k A_{0} \in \mathbb{A}$. Через $\partial_{\text {ext }}(\mathscr{A})$ обозначим минимальное подмножество в $\mathbb{A}_{+}$такое, что

1) $\partial(\mathscr{A}) \subset \partial_{\text {ext }}(\mathscr{A})$;

2) если $X=A_{\nu_{1}}+\cdots+A_{\nu_{q}} \in \partial_{\text {ext }}(\mathscr{A}) \cup\{\mathscr{A}\}$, то $X-A_{\nu_{i}}+A_{\nu_{i}+1} \in \partial_{\text {ext }}(\mathscr{A})$ для любого $i=1, \ldots, q$.

Легко видеть, что

$$
\bigcup_{k \geqslant 0} \partial\left(\mathscr{A}^{k}\right)=\bigcup_{X \in \partial_{\text {ext }}(\mathscr{A})} \bigcup_{k \geqslant 0} X^{k} .
$$

Если $\left(\mathscr{A}^{k}\right)_{f} \neq \emptyset$, то $k \equiv \operatorname{deg} \mathscr{A}+d_{f}(\bmod 2)$. 
Рассмотрим цепи

$$
\begin{aligned}
& \sigma^{0}(f, \mathscr{A})=\sum_{k=0}^{d_{f}-\operatorname{deg} \mathscr{A}}\left(\mathscr{A}^{k}\right)_{f}, \quad \sigma^{1}(f, \mathscr{A})=\sum_{k=0}^{d_{f}-\operatorname{deg} \mathscr{A}} \operatorname{ind}_{f}\left(\mathscr{A}^{k}\right) \cdot\left(\mathscr{A}^{k}\right)_{f}, \\
& R_{c}(f, \mathscr{A})=\sum_{X \in \partial_{\text {ext }}(\mathscr{A}): \operatorname{codim} X=c}\left[\sum_{k=0}^{d_{f}-\operatorname{deg} X} \operatorname{ind}_{f}\left(\mathscr{A}, X^{k}\right) \cdot\left(X^{k}\right)_{f}\right],
\end{aligned}
$$

где

$$
\operatorname{ind}_{f}\left(\mathscr{A}, X^{k}\right)=\sum_{i \geqslant k} \operatorname{ind}_{f}\left(\mathscr{A}^{i}\right) \cdot J_{\mathscr{A}^{i}}\left(X^{k}\right) .
$$

Следующее утверждение является простым упражнением.

ПРЕДЛОЖЕНИЕ 4.2. Для любого $\mathscr{A} \in \mathbb{A}_{+}$

$$
\partial_{*}\left(\sigma^{1}(f, \mathscr{A})\right)=\sum_{c=\operatorname{codim} \mathscr{A}+1}^{n}(-1)^{c} R_{c}(f, \mathscr{A}) .
$$

Из следствия 3.6 и предложения 4.2 получаем.

Теорема 4.3. Пусть $M$ и $N$ - гладкие замкнутые $n$-мерные многообразия, $N$ связно и $f: M \rightarrow N-$ устойчивое гладкое отображсение коранга $\leqslant 1$. Тогда

$$
\sum_{c=\operatorname{codim} \mathscr{A}+1}^{n} \chi\left(R_{c}(f, \mathscr{A})\right) \equiv 0(\bmod 2)
$$

для любого $\mathscr{A} \in \mathbb{A}_{+}($в том числе и для $\mathscr{A}=0)$.

Рассмотрим произвольньй элемент $\mathscr{A}=A_{\mu_{1}}+\cdots+A_{\mu_{p}} \in \mathbb{A}_{+}$. Через \#($\left.A_{\mu} \mid \mathscr{A}\right)$ обозначим число слагаемых в $\mathscr{A}$, равных $A_{\mu}$. Положим

$$
\mathscr{S}_{f}^{0}(\mathscr{A})=\chi\left(\sigma^{0}(f, \mathscr{A})\right), \quad \mathscr{S}_{f}^{1}(\mathscr{A})=\chi\left(\sigma^{1}(f, \mathscr{A})\right)
$$

ЗАмЕчаниЕ 4.4. Число $\mathscr{S}_{f}^{0}(\mathscr{A})$ равно эйлеровой характеристике многообразия особенностей типа $\mathscr{A}$ множества $\mathscr{F}_{f}$ критических значений отображения $f$. Число $\mathscr{S}_{f}^{1}(\mathscr{A})$ сравнимо по модулю 2 с эйлеровой характеристикой многообразия, образованного мультиособенностями типов $\mathscr{A}^{k}$ отображения $f$, где $k+\operatorname{deg} \mathscr{A}+d_{f} \equiv 2(\bmod 4)$.

ПРЕДЛОЖЕНИЕ 4.5. Пусть $\mathscr{A}=\alpha_{1} A_{\mu_{1}}+\cdots+\alpha_{p} A_{\mu_{p}} \in \mathbb{A}_{+}$, əде $\mu_{1}, \ldots, \mu_{p}$ nопарно различны. Тогда

$$
\begin{aligned}
& \chi\left(R_{\operatorname{codim}} \mathscr{A}+1\right. \\
& \quad+\sum_{\alpha_{i}>1}\left[\#\left(A_{2 \mu_{i}+1} \mid \mathscr{A}\right)+1\right] \mathscr{S}_{f}^{1}\left(\mathscr{A}-2 A_{\mu_{i}}+A_{2 \mu_{i}+1}\right)(\bmod 2) \\
& \quad
\end{aligned}
$$


ДоказАТЕЛьство. Элементы $X \in \partial_{\text {ext }}(\mathscr{A})$ такие, что $\operatorname{codim} X=\operatorname{codim} \mathscr{A}+1$, могут быть только следующих трех типов.

1) $X=\mathscr{A}+A_{1}$. В этом случае

$$
\begin{gathered}
\operatorname{ind}_{f}\left(\mathscr{A}, X^{k}\right)=\operatorname{ind}_{f}\left(\mathscr{A}^{k}\right) J_{\mathscr{A}^{k}}\left(X^{k}\right)+\operatorname{ind}_{f}\left(\mathscr{A}^{k+2}\right) J_{\mathscr{A}^{k+2}}\left(X^{k}\right), \\
\operatorname{ind}_{f}\left(\mathscr{A}^{k}\right)=\frac{\operatorname{deg} \mathscr{A}+k+d_{f}}{2}, \quad \operatorname{ind}_{f}\left(\mathscr{A}^{k+2}\right)=\operatorname{ind}_{f}\left(\mathscr{A}^{k}\right)+1, \\
J_{\mathscr{A}^{k}}\left(X^{k}\right)=J_{\mathscr{A}^{k+2}}\left(X^{k}\right)=\#\left(A_{1} \mid \mathscr{A}\right)+1 .
\end{gathered}
$$

Поэтому

$$
\operatorname{ind}_{f}\left(\mathscr{A}, X^{k}\right) \equiv \#\left(A_{1} \mid \mathscr{A}\right)+1(\bmod 2)
$$

2) $X=\mathscr{A}-A_{\mu_{i}}+A_{\mu_{i}+1}$. В этом случае

$$
\begin{gathered}
\operatorname{ind}_{f}\left(\mathscr{A}, X^{k}\right)=\operatorname{ind}_{f}\left(\mathscr{A}^{k+1}\right) J_{\mathscr{A}^{k+1}}\left(X^{k}\right), \\
\operatorname{ind}_{f}\left(\mathscr{A}^{k+1}\right)=\frac{\operatorname{deg} \mathscr{A}+k+1+d_{f}}{2}, \quad J_{\mathscr{A}^{k+1}}\left(X^{k}\right)=2 \cdot\left[\#\left(A_{\mu_{i}+1} \mid \mathscr{A}\right)+1\right] .
\end{gathered}
$$

Поэтому

$$
\operatorname{ind}_{f}\left(\mathscr{A}, X^{k}\right) \equiv 0(\bmod 2) \text {. }
$$

3) $X=\mathscr{A}-A_{\mu_{i}}-A_{\mu_{j}}+A_{\mu_{i}+\mu_{j}+1}$. В этом случае

$$
\operatorname{ind}_{f}\left(\mathscr{A}, X^{k}\right)=\operatorname{ind}_{f}\left(\mathscr{A}^{k}\right) J_{\mathscr{A}^{k}}\left(X^{k}\right), \quad J_{\mathscr{A}^{k}}\left(X^{k}\right)=\delta(i, j) \cdot\left[\#\left(A_{\mu_{i}+\mu_{j}+1} \mid \mathscr{A}\right)+1\right]
$$

где

$$
\delta(i, j)=\left\{\begin{array}{l}
2, \text { если } i \neq j \\
1, \text { если } i=j
\end{array}\right.
$$

Поэтому

$$
\operatorname{ind}_{f}\left(\mathscr{A}, X^{k}\right) \equiv \begin{cases}0(\bmod 2), & \text { если } i \neq j, \\ \frac{\operatorname{deg} \mathscr{A}+k+d_{f}}{2} \cdot\left[\#\left(A_{2 \mu_{i}+1} \mid \mathscr{A}\right)+1\right](\bmod 2), & \text { если } i=j .\end{cases}
$$

Теперь, предложение 4.5 очевидно.

Из теоремы 4.3 и предложения 4.5 получаем.

СлЕДСТВИЕ 4.6. Пусть $M u N$ - гладкие замкнутые $n$-мерные многообразия, $N$ связно и $f: M \rightarrow N$ - устойчивое гладкое отображсение коранга $\leqslant 1$. Рассмотрим әлемент $\mathscr{A}=\alpha_{1} A_{\mu_{1}}+\cdots+\alpha_{p} A_{\mu_{p}} \in \mathbb{A}_{+}$такой, что $\mu_{1}, \ldots, \mu_{p}$ попарно различны, a $\operatorname{codim} \mathscr{A}=n-1$. Тогда

$\left[\#\left(A_{1} \mid \mathscr{A}\right)+1\right] \mathscr{S}_{f}^{0}\left(\mathscr{A}+A_{1}\right) \equiv \sum_{\alpha_{i}>1}\left[\#\left(A_{2 \mu_{i}+1} \mid \mathscr{A}\right)+1\right] \mathscr{S}_{f}^{1}\left(\mathscr{A}-2 A_{\mu_{i}}+A_{2 \mu_{i}+1}\right)(\bmod 2)$ 
Пример 4.7. Пусть $n=1$ и $\mathscr{A}=0$. Тогда из следствия 4.6 получаем $\mathscr{S}_{f}^{0}\left(A_{1}\right) \equiv 0$ $(\bmod 2)$. Это хорошо известно: число критических точек функции Морса на одномерном гладком замкнутом многообразии четно.

Пример 4.8. Пусть $\mathscr{A}=A_{\mu_{1}}+\cdots+A_{\mu_{p}}$, где $\mu_{1}, \ldots, \mu_{p}$ попарно различны, $\mu_{1}>1$, $\ldots, \mu_{p}>1$ и $\mu_{1}+\cdots+\mu_{p}=n-1$. Тогда $\mathscr{S}_{f}^{0}\left(\mathscr{A}+A_{1}\right) \equiv 0(\bmod 2)$. В частности, если $n \geqslant 3$, то

$$
\mathscr{S}_{f}^{0}\left(A_{n-1}+A_{1}\right) \equiv 0(\bmod 2) \text {. }
$$

ПримеР 4.9. Пусть $n \geqslant 3$ нечетное и $\mathscr{A}=(n-1) A_{1}$. Тогда

$$
\mathscr{S}_{f}^{0}\left(n A_{1}\right) \equiv \mathscr{S}_{f}^{1}\left(A_{3}+(n-3) A_{1}\right)(\bmod 2) .
$$

ПримеР 4.10. Пусть $n \geqslant 3$ нечетное и $\mathscr{A}=2 A_{(n-1) / 2}$. Тогда

$$
\mathscr{S}_{f}^{0}\left(2 A_{(n-1) / 2}+A_{1}\right) \equiv \mathscr{S}_{f}^{1}\left(A_{n}\right)(\bmod 2) .
$$

Теорема 4.3 позволяет получить много других утверждений о четности эйлеровых характеристик многообразий мультиособенностей отображения $f$. Мы приведем еще только одно.

ПреДЛОЖенИЕ 4.11. Пусть $\mathscr{A}=\alpha_{1} A_{\mu_{1}}+\cdots+\alpha_{p} A_{\mu_{p}} \in \mathbb{A}_{+}$, где $\mu_{1}, \ldots, \mu_{p}$ nопарно различны. Тогда

$$
\begin{aligned}
& \chi\left(R_{\text {codim } \mathscr{A}+2}(f, \mathscr{A})\right) \equiv\left[\#\left(A_{2} \mid \mathscr{A}\right)+1\right] \mathscr{S}_{f}^{0}\left(\mathscr{A}+A_{2}\right) \\
& +\sum_{\alpha_{i}>1}\left[\#\left(A_{2 \mu_{i}+1} \mid \mathscr{A}\right)+1\right]\left[\#\left(A_{1} \mid \mathscr{A}\right)+1\right] \mathscr{S}_{f}^{0}\left(\mathscr{A}-2 A_{\mu_{i}}+A_{2 \mu_{i}+1}+A_{1}\right) \\
& +\sum_{\alpha_{i}>0}\left[\#\left(A_{\mu_{i}+2} \mid \mathscr{A}\right)+1\right] \mathscr{S}_{f}^{0}\left(\mathscr{A}-A_{\mu_{i}}+A_{\mu_{i}+2}\right) \\
& +\sum_{\alpha_{i}>1}\left[\#\left(A_{2 \mu_{i}+2} \mid \mathscr{A}\right)+1\right] \mathscr{S}_{f}^{1}\left(\mathscr{A}-2 A_{\mu_{i}}+A_{2 \mu_{i}+2}\right) \\
& +\sum_{\alpha_{i}>1, \alpha_{j}>1, j>i}\left[\#\left(A_{2 \mu_{i}+1} \mid \mathscr{A}\right)+1\right]\left[\#\left(A_{2 \mu_{j}+1} \mid \mathscr{A}\right)+1\right] \\
& \times \mathscr{S}_{f}^{1}\left(\mathscr{A}-2 A_{\mu_{i}}-2 A_{\mu_{j}}+A_{2 \mu_{i}+1}+A_{2 \mu_{j}+1}\right) \\
& +\sum_{\alpha_{i}>3}\left(\begin{array}{c}
\#\left(A_{2 \mu_{i}+1} \mid \mathscr{A}\right)+2 \\
2
\end{array}\right) \mathscr{S}_{f}^{1}\left(\mathscr{A}-4 A_{\mu_{i}}+2 A_{2 \mu_{i}+1}\right) \\
& +\sum_{\alpha_{i}>2}\left[\#\left(A_{3 \mu_{i}+2} \mid \mathscr{A}\right)+1\right] \mathscr{S}_{f}^{1}\left(\mathscr{A}-3 A_{\mu_{i}}+A_{3 \mu_{i}+2}\right) \\
& +\sum_{\alpha_{i}>0, \alpha_{j}>1, j \neq i}\left[\#\left(A_{\mu_{i}+2 \mu_{j}+2} \mid \mathscr{A}\right)+1\right] \mathscr{S}_{f}^{1}\left(\mathscr{A}-A_{\mu_{i}}-2 A_{\mu_{j}}+A_{\mu_{i}+2 \mu_{j}+2}\right)
\end{aligned}
$$

по модулю 2.

Доказательство этого утверждения аналогично доказательству предложения 4.5. 
СлЕДСТВИЕ 4.12. Пусть $M$ и $N$ - гладкие замкнутые $n$-мерные многообразия, $N$ связно и $f: M \rightarrow N$ - устойчивое гладкое отображсние коранга $\leqslant 1$. Рассмотрим әлемент $\mathscr{A}=\alpha_{1} A_{\mu_{1}}+\cdots+\alpha_{p} A_{\mu_{p}} \in \mathbb{A}_{+}$такой, что $\mu_{1}, \ldots, \mu_{p}$ попарно различны, a codim $\mathscr{A}=n-2$. Тогда

$$
\begin{aligned}
& {\left[\#\left(A_{1} \mid \mathscr{A}\right)+1\right] \mathscr{S}_{f}^{0}\left(\mathscr{A}+A_{1}\right)} \\
& \quad+\sum_{\alpha_{i}>1}\left[\#\left(A_{2 \mu_{i}+1} \mid \mathscr{A}\right)+1\right] \mathscr{S}_{f}^{1}\left(\mathscr{A}-2 A_{\mu_{i}}+A_{2 \mu_{i}+1}\right) \equiv \chi\left(R_{n}(f, \mathscr{A})\right)
\end{aligned}
$$

по модулю 2 , где $\chi\left(R_{n}(f, \mathscr{A})\right)$ вычисляется согласно предлоэсению 4.11.

ПримеР 4.13. Пусть $n=2$ и $\mathscr{A}=0$. Тогда из следствия 4.12 получаем $\mathscr{S}_{f}^{0}\left(A_{1}\right) \equiv$ $\mathscr{S}_{f}^{0}\left(A_{2}\right)(\bmod 2)$.

ПримеР 4.14. Пусть $n=3$ и $\mathscr{A}=A_{1}$. Тогда из следствия 4.12 и примера 4.8 получаем $\mathscr{S}_{f}^{0}\left(A_{3}\right) \equiv 0(\bmod 2)$. Это хорошо известно: число ласточкиных хвостов на компактном волновом фронте общего положения в трехмерном пространстве четно.

Пример 4.15. Пусть $n=4$ и $\mathscr{A}=A_{2}$. Тогда $\mathscr{S}_{f}^{0}\left(A_{2}+A_{1}\right) \equiv \mathscr{S}_{f}^{0}\left(A_{4}\right)(\bmod 2)$.

ПримеР 4.16. Пусть $n \geqslant 5$ и $\mathscr{A}=A_{n-2}$. Тогда

$$
\mathscr{S}_{f}^{0}\left(A_{n-2}+A_{1}\right) \equiv \mathscr{S}_{f}^{0}\left(A_{n}\right)+\mathscr{S}_{f}^{0}\left(A_{n-2}+A_{2}\right)(\bmod 2) .
$$

В малых размерностях можно перечислить все сравнения, которые следуют из теоремы 4.3.

ПРЕДЛОЖЕНИЕ 4.17. Справедливы следующие сравнения по модулю 2:

$$
\begin{aligned}
\chi\left(R_{3}(f, 0)\right) & \equiv \mathscr{S}_{f}^{0}\left(A_{3}\right)+\mathscr{S}_{f}^{1}\left(A_{3}\right), \\
\chi\left(R_{4}(f, 0)\right) & \equiv \mathscr{S}_{f}^{0}\left(A_{3}+A_{1}\right)+\mathscr{S}_{f}^{0}\left(A_{4}\right)+\mathscr{S}_{f}^{1}\left(A_{4}\right), \\
\chi\left(R_{4}\left(f, A_{1}\right)\right) & \equiv \mathscr{S}_{f}^{0}\left(A_{3}+A_{1}\right)+\mathscr{S}_{f}^{1}\left(A_{3}+A_{1}\right) .
\end{aligned}
$$

Доказательство заключается в непосредственном вычислении.

СлЕДСТВИЕ 4.18. Пусть $M$ и $N$ - гладкие замкнутые $n$-мерные многообразия, $N$ связно и $f: M \rightarrow N$ - устойчивое гладкое отображение коранга $\leqslant 1$. Тогда имеют место следующие сравнения по модулю 2:

\begin{tabular}{|c|c|}
\hline$n=1$ & $\mathscr{S}_{f}^{0}\left(A_{1}\right) \equiv 0$ \\
\hline$n=2$ & $\mathscr{S}_{f}^{0}\left(A_{1}\right) \equiv \mathscr{S}_{f}^{0}\left(A_{2}\right)$ \\
\hline \multirow{3}{*}{$n=3$} & $\mathscr{S}_{f}^{0}\left(A_{1}\right) \equiv \mathscr{S}_{f}^{0}\left(A_{2}\right)+\mathscr{S}_{f}^{1}\left(A_{3}\right)$ \\
\cline { 2 - 3 } & $\mathscr{S}_{f}^{0}\left(3 A_{1}\right) \equiv \mathscr{S}_{f}^{1}\left(A_{3}\right)$ \\
\cline { 2 - 3 } & $\mathscr{S}_{f}^{0}\left(A_{2}+A_{1}\right) \equiv \mathscr{S}_{f}^{0}\left(A_{3}\right) \equiv 0$ \\
\hline \multirow{3}{*}{$n=4$} & $\mathscr{S}_{f}^{0}\left(A_{1}\right) \equiv \mathscr{S}_{f}^{0}\left(A_{2}\right)+\mathscr{S}_{f}^{1}\left(A_{3}\right)+\mathscr{S}_{f}^{1}\left(A_{4}\right)$ \\
\cline { 2 - 2 } & $\mathscr{S}_{f}^{0}\left(3 A_{1}\right)+\mathscr{S}_{f}^{0}\left(A_{2}+2 A_{1}\right) \equiv \mathscr{S}_{f}^{1}\left(A_{3}\right)+\mathscr{S}_{f}^{1}\left(A_{4}\right)$ \\
\cline { 2 - 2 } & $\mathscr{S}_{f}^{0}\left(A_{2}+A_{1}\right) \equiv \mathscr{S}_{f}^{0}\left(A_{3}\right) \equiv \mathscr{S}_{f}^{0}\left(A_{4}\right)$ \\
\cline { 2 - 2 } & $\mathscr{S}_{f}^{0}\left(A_{3}+A_{1}\right) \equiv \mathscr{S}_{f}^{1}\left(A_{3}+A_{1}\right) \equiv 0$ \\
\hline
\end{tabular}


ЗАмЕчАниЕ 4.19. Теорема 4.3 не дает всех ограничений на четность эйлеровых характеристик многообразий мультиособенностей отображения $f$. Например, если $n=3$, то $\mathscr{S}_{f}^{0}\left(A_{2}\right)=\mathscr{S}_{f}^{0}\left(A_{2}+A_{1}\right)+\mathscr{S}_{f}^{0}\left(A_{3}\right)$ (см. вторую формулу таблицы 1 из [7]). Отсюда следует сравнение $\mathscr{S}_{f}^{0}\left(A_{2}\right) \equiv 0(\bmod 2)$, которое нельзя получить только из следствия 4.18.

5. Приложения к пространственным кривым. Рассмотрим произвольную гладкую замкнутую кривую $\gamma$ общего положения в $\mathbb{R} P^{n}$. Ее касательные гиперплоскости классифицируются по ненулевым элементам полугрупшы $\mathbb{A}_{+}$(свободной абелевой полугруппы по сложению, порожденной символами $\left.A_{1}, A_{2}, \ldots, A_{\mu}, \ldots\right)$.

А именно, пусть $\pi \subset \mathbb{R} P^{n}$ - касательная гиперплоскость кривой $\gamma$. Рассмотрим произвольный элемент $\mathscr{A}=A_{\mu_{1}}+\cdots+A_{\mu_{p}} \neq 0$ полугрупшы $\mathbb{A}_{+} \cdot$ Напомним, что $\operatorname{codim} \mathscr{A}=\mu_{1}+\cdots+\mu_{p}, \operatorname{deg} \mathscr{A}=\operatorname{codim} \mathscr{A}+p$ и \# $\left(A_{\mu} \mid \mathscr{A}\right)$ - число слагаемых в $\mathscr{A}$, равных $A_{\mu}$.

ОПРЕДЕЛЕНИЕ 5.1. ГИперплоскость $\pi$ называется касательной әиперплоскостью типа $\mathscr{A}$, если

1) она касается кривой $\gamma$ ровно в $p$ попарно различных точках с кратностями $\mu_{1}$, $\ldots, \mu_{p}$, соответственно;

2) эти точки являются вершинами $(p-1)$-мерного симплекса.

Касательные гиперплоскости кривой $\gamma$ общего положения исчерпываются касательными гиперплоскостями типов $\mathscr{A} \in \mathbb{A}_{+} \backslash\{0\}$ с коразмерностью касания $\operatorname{codim} \mathscr{A} \leqslant n$ (см. [4], [12]). Для любого $\mathscr{A}$ такого, что $\operatorname{codim} \mathscr{A}=n$, число $\mathscr{S}_{\gamma}^{0}(\mathscr{A})$ всех касательных гиперплоскостей типа $\mathscr{A}$ кривой $\gamma$ конечно. Обозначим через $\mathscr{S}_{\gamma}^{1}(\mathscr{A})$ число касательных гиперплоскостей типа $\mathscr{A}$, у каждой из которых число точек трансверсального пересечения с $\gamma$ сравнимо с $2-\operatorname{deg} \mathscr{A}-\theta(\gamma)$ по модулю 4 , где $\theta(\gamma)$ - число нестягиваемых компонент кривой $\gamma$.

Tеорема 5.2. Пусть $\gamma-$ гладкая замкнутая кривая в $\mathbb{R} P^{n}$. Рассмотрим әлемент $\mathscr{A}=\alpha_{1} A_{\mu_{1}}+\cdots+\alpha_{p} A_{\mu_{p}} \in \mathbb{A}_{+} \backslash\{0\}$ такой, что $\mu_{1}, \ldots, \mu_{p}$ попарно различны, $a \operatorname{codim} \mathscr{A}=n-1$. Тогда для кривой $\gamma$ общего положения

$$
\left[\#\left(A_{1} \mid \mathscr{A}\right)+1\right] \mathscr{S}_{\gamma}^{0}\left(\mathscr{A}+A_{1}\right) \equiv \sum_{\alpha_{i}>1}\left[\#\left(A_{2 \mu_{i}+1} \mid \mathscr{A}\right)+1\right] \mathscr{S}_{\gamma}^{1}\left(\mathscr{A}-2 A_{\mu_{i}}+A_{2 \mu_{i}+1}\right)
$$

по модулю 2.

ДокАЗАтЕльство. Рассмотрим проективизацию $P T^{*}\left(\mathbb{R} P^{n}\right)$ кокасательного расслоения пространства $\mathbb{R} P^{n}$. Это гладкое $(2 n-1)$-мерное многообразие. Оно образовано контактными элементами $(\pi, x)$, где $x \in \mathbb{R} P^{n}$, а $\pi \in \mathbb{R} P^{n *}-$ гиперплоскость в $\mathbb{R} P^{n}$, проходящая через точку $x$.

Многообразие $P T^{*}\left(\mathbb{R} P^{n}\right)$ имеет естественную контактную структуру (см. [4]). Проекция

$$
\varrho: P T^{*}\left(\mathbb{R} P^{n}\right) \rightarrow \mathbb{R} P^{n *}, \quad(\pi, x) \mapsto \pi,
$$

является лежандровым расслоением относительно этой структуры.

Рассмотрим множество $M$ контактньх элементов $(\pi, x)$, где $x \in \gamma$. Это множество является гладким замкнутым $n$-мерньп подмногообразием в $P T^{*}\left(\mathbb{R} P^{n}\right)$. Ограничение проекции $\varrho$ на многообразие $M$ является гладким отображением $f: M \rightarrow \mathbb{R} P^{n *}$. 
Множество $L_{f}$ критических точек отображения $f$ состоит из контактных элементов $(\pi, x) \in P T^{*}\left(\mathbb{R} P^{n}\right)$, где $\pi-$ касательная гиперплоскость кривой $\gamma$ в точке $x$. Множество $\mathscr{F}_{f}$ критических значений этого отображения состоит из гиперплоскостей в $\mathbb{R} P^{n}$, касающихся кривой $\gamma$.

Для кривой $\gamma$ общего положения множество $L_{f}$ является гладким $(n-1)$-мерным лежандровьм подмногообразием в $P T^{*}\left(\mathbb{R} P^{n}\right)$. Множество $\mathscr{F}_{f}$ является фронтом в $\mathbb{R} P^{n *}$ (а именно, проекцией многообразия $L_{f}$ в базу расслоения $\varrho$ ). При этом фронт $\mathscr{F} f$ устойчив и имеет особенности только коранга 1. Для любого $\mathscr{A} \in \mathbb{A}_{+} \backslash\{0\}$ особенности типа $\mathscr{A}$ фронта $\mathscr{F}_{f}$ взаимно однозначно соответствуют касательным гиперплоскостям типа $\mathscr{A}$ кривой $\gamma$ (см. [4], [12]).

Следовательно, для кривой $\gamma$ общего положения отображение $f$ является устойчивым отображением коранга $\leqslant 1$. Максимум $d_{f}$ числа точек в полном прообразе $f^{-1}(\pi)$ по всем $\pi \in \mathbb{R} P^{n *}$ сравним по модулю 2 с числом $\theta(\gamma)$. Для любого $\mathscr{A} \in \mathbb{A}_{+} \backslash\{0\}$ касательные гиперплоскости типа $\mathscr{A}$ кривой $\gamma$ взаимно однозначно соответствуют мультиособенностям типов $\mathscr{A}+k A_{0} \in \mathbb{A}$ отображения $f$. В частности, $\mathscr{S}_{f}^{i}(\mathscr{A})=\mathscr{S}_{\gamma}^{i}(\mathscr{A})$ для любого $i=0,1$ и для любого $\mathscr{A}$ такого, что codim $\mathscr{A}=n$. Теперь теорема 5.2 вытекает из следствия 4.6 (и замечания 4.4 ).

Из теоремы 5.2 следуют теоремы 1.2 и 1.3 (см. примеры 4.9 и 4.10). Из нее следуют также и некоторые другие факты о четности чисел гиперплоскостей различных типов, касающихся с максимально возможной коразмерностью гладкой замкнутой кривой общего положения в $\mathbb{R} P^{n}$. В частности (см. пример 4.8), имеет место следующее утверждение.

ТеОрема 5.3. Пусть $\gamma-$ гладкая замкнутая кривая в $\mathbb{R} P^{n}$. Предположим, что $\mu_{1}, \ldots, \mu_{p}-$ попарно различные челые числа, $\mu_{1}>1, \ldots, \mu_{p}>1 u \mu_{1}+\cdots+\mu_{p}=n-1$. Тогда при любом $n \neq 2$ число касательных гиперплоскостей типа $A_{\mu_{1}}+\cdots+$ $A_{\mu_{p}}+A_{1}$ кривой $\gamma$ общего полохсения четно.

Рассмотрим, например, соприкасающиеся гиперплоскости гладкой замкнутой кривой $\gamma$ в $\mathbb{R} P^{n}$. Соприкасающаяся гиперплоскость в общей точке кривой $\gamma$ общего положения касается кривой только в этой точке с кратностью $n-1$. Соприкасающиеся гиперплоскости в некоторых изолированных точках могут касаться кривой $\gamma$ с коразмерностью $n$. Эти соприкасающиеся гиперплоскости называются особыми и являются касательньми гиперплоскостями двух типов: $A_{n}$ и $A_{n-1}+A_{1}$. Поскольку $\mathscr{S}_{\gamma}^{0}\left(A_{n}\right) \equiv(n+1) \theta(\gamma)(\bmod 2)$, из теоремы 5.3 получаем

СлЕДСТВИЕ 5.4. Число особых соприкасающихся гиперплоскостей гладкой замкнутой кривой $\gamma$ общего положсения в $\mathbb{R} P^{n}$ сравнимо $c(n+1) \theta(\gamma)$ по модулю 2 для любого $n \geqslant 3$.

В случае $n=3$ этот факт указан также в [13].

Статья была частично написана в университете г. Утрехта (Нидерланды). Автор благодарен Д. Сирсме и всем сотрудникам математического факультета этого университета за гостеприимство и поддержку. Я хотел бы также поблагодарить М.Э. Казаряна за полезные обсуждения и замечания. 


\section{СПИСОК ЦИТИРОВАННОЙ ЛИТЕРАТУРЫ}

[1] Banchoff Th., Gaffney T., McCrory C. Counting tritangent planes of space curves // Topology. 1985. V. 24. №1. P. 15-23.

[2] Freedman M.H. Planes triply tangent to curves with nonvanishing torsion // Topology. 1980. V. 19. № 1. P. 1-8.

[3] Morin B. Formes canoniques des singularités d'une application différentiable // C. R. Acad. Sci. Paris. 1965. V. 260. P. 5662-5665.

[4] Арнольд В.И., Варченко А. Н., Гусейн-Заде С. М. Особенности дифференцируемых отображений. Т. 1. М.: Наука, 1982.

[5] Васильев В.А. Лагранжевы и лежандровы характеристические классы. М.: МЦНМО, 2000.

[6] Kazarian M. E. Thom polynomials for Lagrange, Legendre, and critical point function singularities // Proc. London Math. Soc. 2003. V. 86. № 3. P. 707-734.

[7] Седых В. Д. Разрешение особенностей коранга 1 фронта общего положения // Функцион. анализ и его прилож. 2003. Т. 37. № 2. С. 52-64.

[8] McCrory C., Parusiński A. Algebraically constructible functions // Ann. Sci. 'Ecole Norm. Sup. 1997. V. 30. № 4. P. 527-552.

[9] Мазер Дж. Стратификации и отображения // УМН. 1972. Т. 27. № 5. С. 85-118.

[10] Romero Fuster M. C. Sphere stratifications and the Gauss map // Proc. Roy. Soc. Edinburgh. Sect. A. 1983. V. 95. P. 115-136.

[11] Седых В. Д. О топологии образа устойчивого гладкого отображения с особенностями коранга 1 // Докл. РАН. 2004. Т. 395. № 4. С. 459-463.

[12] Седых В. Д. Строение выпуклой оболочки пространственной кривой // Тр. сем. им. Петровского. 1981. Т. 6. С. 239-256.

[13] Седых В. Д. Двойные касательные плоскости к пространственной кривой // Сиб. матем. ж. 1989. Т. 30. № 1. С. 209-211.

Российский государственный университет

Поступило

нефьти и газа им. И. М. Губкина

21.10 .2003

E-mail : sedykh@mccme.ru

Исправленный вариант

08.02 .2005 